\title{
Melanoma Cutâneo em um Hospital Universitário, 2001-2016
}

doi: https://doi.org/10.32635/2176-9745.RBC.2020v66n3.911

\author{
Cutaneous Melanoma in a University Hospital, 2001-2016 \\ Melanoma Cutáneo en un Hospital Universitario, 2001-2016
}

\author{
Mariana Barufaldi Bertoldi'; Carlos Augusto Silva Bastos²; Carolina Labigalini Sampaio ${ }^{3}$; Eduardo Henrique Wroblevski"; Kátia \\ Sheylla Malta Purim ${ }^{5}$
}

\begin{abstract}
RESUMO
Introduçáo: O melanoma é a principal causa de morte entre as neoplasias malignas cutâneas primárias. Sua incidência mundial vem aumentando progressivamente, entretanto, existem escassas informações epidemiológicas nacionais. Objetivo: Analisar os perfis epidemiológico e histopatológico de melanomas cutâneos diagnosticados em hospital universitário nos últimos 16 anos. Método: Série histórica de casos de melanoma cutâneo realizada por meio da revisão de prontuários e laudos histopatológicos de 2001 a 2016 . Resultados: A frequência manteve-se com média de 2,99 melanomas por mil novos atendimentos ambulatoriais. A casuística foi de 224 melanomas cutâneos em 211 pacientes, brancos (98,6\%), mulheres (55,9\%), com idade média de 57,3 anos. O tempo médio entre o aparecimento da lesão (desde surgimento da lesão ou desde que a lesão começou a se modificar, relatado pelo paciente) e o diagnóstico foi 4,8 anos. $\mathrm{O}$ tamanho predominante foi de 0,5 a $2 \mathrm{~cm}$, acometendo principalmente a região cefálica em indivíduos maiores de 60 anos e troncular naqueles menores de 60 anos. "Outros melanomas" (34,8\%) e melanoma extensivo superficial $(31,7 \%)$ foram os subtipos mais frequentes. A maioria dos casos apresentou índice de Breslow $\leq 1 \mathrm{~mm}(70 \%)$. Os subtipos mais finos $(\leq 1 \mathrm{~mm})$ foram extensivo superficial e lentigo maligno. Melanomas nodulares possuíam Breslow intermediário (1 a $4 \mathrm{~mm}$ ) ou espesso $(\geq 4 \mathrm{~mm})$ com altas taxas de disseminação e metástase linfonodal. Conclusáo: A frequência manteve-se estável. Houve prevalência em população maior de 60 anos. Os subtipos mais frequentes foram "outros melanomas" e extensivo superficial com localizaçáo cefálica e troncular em sua maioria.
\end{abstract}

Palavras-chave: Melanoma/epidemiologia; Neoplasias Cutâneas/epidemiologia; Dermatopatias.

\section{ABSTRACT}

Introduction: Melanoma is the main cause of death among primary skin neoplasms. The worldwide incidence has been increasing, however there is scarce national epidemiological information. Objective: Analyze the epidemiological and histopathological profile of cutaneous melanomas diagnosed at a university hospital in the last 16 years. Method: Historical series of cutaneous melanoma cases from review of charts and histopathological reports from 2001 to 2016. Results: The mean frequency over the years was 2.99 melanomas for every 1,000 new outpatient visits. The casuistry consisted of 224 cases of cutaneous melanomas in 211 patients, Caucasians $(98.6 \%)$, women $(55.9 \%)$, with mean age of 57.3 years. The mean time between onset of the lesion (time since the appearance of the lesion or since it began to change reported by the patient) and diagnosis was 4.8 years. The predominant tumor size was 0.5 to $2 \mathrm{~cm}$, affecting the cephalic region in individuals older than 60 years and torso in those younger than 60 years. "Other melanomas" (34.8\%) and superficial spreading melanoma $(31.7 \%)$ were the predominant subtypes. Most of the cases had Breslow index $\leq 1 \mathrm{~mm}$ ( $70 \%$ of patients). The thinnest subtypes $(\leq 1 \mathrm{~mm})$ were superficial spreading melanoma and lentigo maligna. Nodular melanomas had intermediate Breslow (1 to $4 \mathrm{~mm}$ ) or thick ( $\geq 4 \mathrm{~mm}$ ) with high dissemination rate and lymph node metastasis. Conclusion: The frequency remained stable. Population below 60 years old had a higher prevalence. The most frequent subtypes were "other melanomas" and extensive superficial located mainly in the cephalic and torso regions.

Key words: Melanoma/epidemiology; Skin Neoplasms/epidemiology; Skin Diseases.

\section{RESUMEN}

Introduçáo: El melanoma es la principal causa de muerte entre las neoplasias malignas cutáneas primarias. Su incidencia mundial ha aumentado progresivamente, sin embargo, la información epidemiológica nacional es escasa. Objetivo: Analizar el perfil epidemiológico e histopatológico de los melanomas cutáneos diagnosticados en un hospital universitario en 16 años. Método: Serie histórica de casos de melanoma cutáneo obtenidos por la revisión de registros médicos e informes histopatológicos de 2001-2016. Resultados: La frecuencia se mantuvo con un promedio de 2,99 melanomas/ mil nuevas consultas. La casuística consistió en 224 melanomas cutáneos en 211 pacientes, blancos $(98,6 \%)$, mujeres $(55,9 \%)$, con edad media de 57,3 años. El promedio entre la aparición de la lesión (desde la aparición o desde que comenzó a cambiar según informe del paciente) y diagnóstico fue 4,8 ańos. El tamańo predominante de 0,5 a $2 \mathrm{~cm}$, afectando principalmente la región cefálica en mayores de 60 años y el tronco cuando menores de 60. "Otros melanomas" $(34,8 \%)$ y melanoma extenso superficial $(31,7 \%)$ fueron los subtipos más frecuentes. La mayoría de los casos tenía índice de Breslow $\leq 1 \mathrm{~mm}(70 \%)$. Los más delgados $(\leq 1 \mathrm{~mm})$ fueron lentigo extenso superficial y maligno. Los nodulares tenían Breslow intermedio (1 a 4 $\mathrm{mm}$ ) o grueso $(\geq 4 \mathrm{~mm})$ con altas tasas de diseminación y metástasis para ganglios linfáticos. Conclusión: La frecuencia se mantuvo estable. Hubo mayor prevalencia en la población menor de 60 ańos. Los subtipos más frecuentes fueron "otros melanomas" y extensos superficiales con localización principalmente cefálica y del tronco.

Palabras clave: Melanoma/epidemiología; Neoplasias Cutáneas/ epidemiología; Enfermedades de la Piel.

\footnotetext{
${ }^{1}$ Departamento de Clínica Médica. Ambulatório de Dermatologia do Complexo Hospital de Clínicas da Universidade Federal do Paraná (UFPR). Curitiba (PR), Brasil. Orcid iD: https://orcid.org/0000-0003-2565-1662

${ }^{2}$ Departamento de Clínica Médica. Ambulatório de Dermatologia do Complexo Hospital de Clínicas da UFPR. Curitiba (PR), Brasil. Orcid iD: https://orcid. org/0000-0003-0255-2696

${ }^{3}$ UFPR. Curitiba (PR), Brasil. Orcid iD: https://orcid.org/0000-0001-6932-9240

${ }^{4}$ UFPR. Curitiba (PR), Brasil. Orcid iD: https://orcid.org/0000-0002-8790-5456

${ }^{5}$ Departamento de Clínica Médica. Ambulatório de Dermatologia do Complexo Hospital de Clínicas da UFPR. Curitiba (PR), Brasil. Orcid iD: https://orcid. org/0000-0001-9982-6408

Endereço para correspondência: Carolina Labigalini Sampaio. Avenida Silva Jardim, 1618 - Rebouças. Curitiba (PR), Brasil. CEP 80240-020. E-mail: carolinalabigalinisampaio@gmail.com
} 


\section{INTRODUÇÃO}

O melanoma é uma neoplasia maligna dos melanócitos com elevada capacidade de disseminação e óbito. Apesar de pouco frequente, é um dos cânceres mais comuns em jovens e relevante causa de morbidade e mortalidade. Configura problema de saúde pública, porém os dados brasileiros ainda são escassos ${ }^{1,2}$.

Segundo análises recentes do Instituto Nacional de Câncer José de Alencar Gomes da Silva (INCA), estimam-se 3,94 novos casos de melanoma para cada $100 \mathrm{mil}$ mulheres e 4,03 novos casos para cada 100 mil homens ${ }^{3}$. O melanoma representa apenas de 3-4\% dos tumores cutâneos malignos no país, mas é responsável por $65-80 \%$ dos óbitos causados por câncer de pele $e^{4,5}$. Os fatores de risco para o desenvolvimento da neoplasia são histórico pessoal e familiar de melanoma, fototipo baixo, doenças genéticas de deficiência no reparo do DNA, exposição solar intensa, residência em latitudes equatoriais ${ }^{6}$, exposição à radiação ultravioleta, imunossupressão, nevos melanocíticos adquiridos e atípicos ${ }^{7}$. Austrália e Nova Zelândia têm as maiores incidências mundiais de melanomas provavelmente em virtude de questōes étnicas e geoclimáticas ${ }^{6,8}$.

Os principais subtipos por ordem de prevalência são o extensivo superficial, nodular, lentigo maligno e lentiginoso acral ${ }^{2,6}$. Formas menos comuns são os subtipos amelanótico, spitzoide, desmoplásico, melanoma de partes moles, nevo azul maligno, ocular e melanoma mucoso ${ }^{5,6}$.

Achados clínicos como mudança da cor, forma, tamanho de uma lesão pigmentada durante um período, cursando ou náo com sangramento, prurido, dor ou ulceração chamam atenção. $\mathrm{O}$ exame dermatológico adequado com dermatoscopia é fundamental para o diagnóstico do melanoma. A Campanha Nacional de Prevenção ao Câncer de Pele ocorre anualmente no Brasil, sendo importante ferramenta para a detecção precoce desses tumores ${ }^{9}$. Toda lesão suspeita deve ser submetida à biopsia excisional e a histopatologia é o padrão-ouro para o diagnóstico ${ }^{6}$. A espessura do tumor (índice de Breslow) é o mais forte preditor de prognóstico e sobrevida ${ }^{2,4-7}$.

A classificação mais utilizada atualmente é a do estadiamento TNM, revisada periodicamente pelo American Joint Comittee on Cancer (AJCC), em que o "T" corresponde ao tamanho do tumor medido pelo Breslow (espessura medida da camada granulosa até a última célula tumoral), "N", ao acometimento linfonodal e "M", às metástases a distância. As mitoses do componente intradérmico e a presença de ulceração são consideradas critérios independentes para morbimortalidade ${ }^{6,10}$.

O prognóstico dos pacientes com melanoma depende do estágio e do diagnóstico. Para pacientes com melanoma cutâneo fino e localizado, geralmente é favorável, sendo que diagnósticos mais precoces contribuem para menor mortalidade proporcional ${ }^{4,9}$.

O objetivo deste estudo foi analisar o perfil epidemiológico e histopatológico dos casos de melanomas cutâneos diagnosticados entre 2001 a 2016 em um hospital público de Curitiba-PR, Brasil, visto sua importância e impactos. Conhecer as características e o comportamento regional do melanoma auxilia no melhor entendimento da doença e contribui para o diagnóstico, seguimento e tratamento do paciente.

\section{MÉTODO}

Foi realizada análise retrospectiva de dados do banco de câncer do hospital e prontuários eletrônicos (onde há dados referentes a laudos anatomopatológicos e exames complementares laboratoriais e de imagem) dos casos de melanoma diagnosticados em um hospital universitário no período de janeiro de 2001 a dezembro de 2016. O projeto foi aprovado pelo Comitê de Ética em Pesquisa institucional sob o número CAAE: 78930717.0.0000.0096 e seguiu a Resolução número 466/2012.

Os critérios de inclusão foram indivíduos de qualquer faixa etária, cor/raça/etnia/sexo com diagnóstico de melanoma cutâneo primário confirmado por histopatologia no período e disponibilidade de prontuário. Foram excluídos prontuários incompletos.

As variáveis analisadas foram sexo, idade, raça dos pacientes, cidade de origem, frequência dos casos no ambulatório de Dermatologia do Complexo Hospital de Clínicas da Universidade Federal do Paraná ao longo dos anos, topografia das lesões, tamanho, sintomatologia, tempo de evolução, método cirúrgico para diagnóstico, tipo histológico, índice de Breslow, presença de metástases, estadiamento e tratamento.

Os dados foram coletados a partir de prontuário eletrônico e do registro hospitalar de câncer. É conduta habitual do Serviço onde o estudo foi feito realizar a dermatoscopia de todas as lesóes suspeitas, porém, como os prontuários físicos dos pacientes não foram acessados, não se utilizaram dados desse recurso diagnóstico.

Para fins deste estudo, os casos foram agrupados em: melanoma extensivo superficial, lentigo maligno, melanoma nodular, melanoma acral e "outros melanomas" que compreendem os subtipos menos comuns (amelanótico, desmoplásico, spitzoide, associados a nevos, entre outros) e os melanomas que não possuíam subespecificação no laudo anatomopatológico. Os tumores foram classificados por meio do Breslow como finos $(\leq 1$ $\mathrm{mm})$, intermediários (1 a $4 \mathrm{~mm}$ ) e espessos ( $\geq 4 \mathrm{~mm})$. 
Os resultados de variáveis quantitativas foram descritos por médias, desvios-padrôes, valores mínimos e máximos. Para variáveis categóricas, foram apresentadas as frequências e percentuais. Para avaliar a associação entre duas variáveis quantitativas, foi considerado o teste de qui-quadrado. Valores de $\mathrm{p}<0,05$ indicaram significância estatística. Para cada uma das variáveis, as análises foram feitas considerando-se os dados válidos (não perdidos). Estes foram analisados com o programa computacional Stata/SE v.14.1. StataCorpLP, USA.

\section{RESULTADOS}

Um total de 211 pacientes com diagnóstico de melanoma cutâneo foi atendido no período de 2001 a 2016. O número de tumores foi de 224 , em razáo de pacientes que apresentaram mais de uma lesáo. A frequência de diagnóstico de melanoma entre os anos se manteve estável nesse Serviço de Dermatologia, com pico em 2008 e média de 2,99 casos para cada mil atendimentos ambulatoriais novos. Esses dados referem-se aos anos de 2005 a 2014 pela náo disponibilidade de informaçóes sobre o número de pacientes atendidos no ambulatório nos demais anos (Gráfico 1).

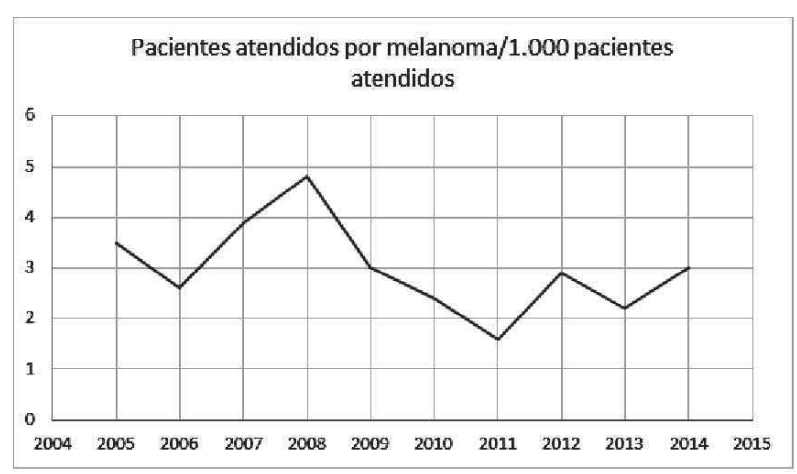

Gráfico 1. Casos de melanoma ao longo de dez anos do ambulatório de Dermatologia

A idade média dos pacientes foi de 57,3 anos com desvio-padrão de 15,6 anos, sendo o indivíduo mais novo de 12 anos e o indivíduo mais velho com 90 anos. Destes, 53,6\% estavam abaixo dos 60 anos. Houve maior participação de mulheres $(55,9 \%)$, brancas $(98,6 \%)$, procedentes de Curitiba e Regiáo metropolitana $(84,8 \%)$. Apenas nove pacientes tiveram dois ou mais melanomas cutâneos (Tabela 1).

Em relação às lesões, o tempo médio de evolução (surgimento da lesão ou modificação da doença) até o diagnóstico foi de 4,8 anos, com localização cefálica $(34,6 \%)$ ou troncular $(27,1 \%)$ sendo as mais comuns. $\mathrm{O}$ tamanho do melanoma predominou entre 0,5 a $2 \mathrm{~cm}$
Tabela 1. Variáveis relativas aos pacientes com diagnóstico de melanoma cutâneo, 2001-2016

\begin{tabular}{|c|c|c|c|}
\hline Variável & $\begin{array}{l}\text { Dados } \\
\text { válidos }\end{array}$ & Classificação & $\begin{array}{c}\text { Resultado* } \\
\text { (\%) }\end{array}$ \\
\hline Idade & 211 & (anos) & $\begin{array}{c}57,3 \pm 15,6 \\
(12-90)\end{array}$ \\
\hline \multirow{2}{*}{ Idade } & \multirow{2}{*}{211} & $<60$ anos & $113(53,6)$ \\
\hline & & $\geq 60$ anos & $98(46,4)$ \\
\hline \multirow{2}{*}{ Sexo } & \multirow{2}{*}{211} & Masculino & $93(44,1)$ \\
\hline & & Feminino & $118(55,9)$ \\
\hline \multirow{3}{*}{ Raça/Cor } & \multirow{3}{*}{209} & Branco & $206(98,6)$ \\
\hline & & Preta & $2(0,9)$ \\
\hline & & Parda & $1(0,5)$ \\
\hline \multirow{2}{*}{ Profissão } & \multirow{2}{*}{85} & Não trabalha & $35(41,2)$ \\
\hline & & Trabalha & $50(58,8)$ \\
\hline \multirow{5}{*}{ Origem } & \multirow{5}{*}{210} & Curitiba & $128(61,0)$ \\
\hline & & $\begin{array}{l}\text { Região } \\
\text { metropolitana }\end{array}$ & $50(23,8)$ \\
\hline & & Interior & $26(12,4)$ \\
\hline & & Litoral & $5(2,4)$ \\
\hline & & Outro Estado & $1(0,5)$ \\
\hline \multirow{3}{*}{ Etilismo } & \multirow{3}{*}{191} & Não & $162(84,8)$ \\
\hline & & Sim & $20(10,5)$ \\
\hline & & $\begin{array}{l}\text { Ex- } \\
\text {-consumidor }\end{array}$ & $9(4,7)$ \\
\hline \multirow{3}{*}{ Tabagismo } & \multirow{3}{*}{198} & Sim & $52(26,3)$ \\
\hline & & Não & $118(59,6)$ \\
\hline & & $\begin{array}{l}\text { Ex- } \\
\text {-consumidor }\end{array}$ & $28(14,1)$ \\
\hline \multirow{2}{*}{$\begin{array}{l}\text { Câncer na } \\
\text { família }\end{array}$} & \multirow{2}{*}{183} & Sim & $90(49,2)$ \\
\hline & & Não & $93(50,8)$ \\
\hline
\end{tabular}

Legenda: ${ }^{*}$ Descrito por média \pm desvio-padrăo (mínimo - máximo) ou por frequência (percentual).

$(74,8 \%)$ e apenas $6,6 \%$ eram $<5 \mathrm{~mm}$. O subtipo extensivo superficial foi o mais frequentemente encontrado no estudo. A frequência de cada subtipo de melanoma, incluindo os melanomas do grupo "outros melanomas", está especificada na Tabela 2.

Cinquenta por cento dos tumores eram restritos à epiderme e $70,2 \%$ foram classificados como melanomas finos $(\leq 1 \mathrm{~mm})$. No entanto, $13,1 \%$ tinham índice de Breslow $\geq 4 \mathrm{~mm}$. Quando analisados os subtipos, verificou-se que $83 \%$ dos extensivos superficiais e $91 \%$ dos lentigos malignos tinham menos que $1 \mathrm{~mm}$ de espessura. Em contrapartida, $100 \%$ dos melanomas nodulares já foram classificados como intermediários ou espessos (>1 mm)

A cirurgia foi o tratamento único de escolha em 174 casos $(77,7 \%)$. Naqueles que necessitaram de terapia 
adjuvante, a quimioterapia foi indicada em 12 (5,4\%) e a radioterapia em sete $(3,1 \%)$. Sete pacientes fizeram tratamentos combinados.

Tabela 2. Subtipo específico de melanoma cutâneo

\begin{tabular}{lc}
\hline \multicolumn{1}{c}{$\begin{array}{c}\text { Subtipos de melanoma cutâneo } \\
(\mathbf{n = 2 2 4 )}\end{array}$} & $\mathbf{n}(\%)$ \\
\hline Melanoma extensivo superficial & $71(31,7)$ \\
Outros melanomas & $61(27,2)$ \\
Lentigo maligno & $27(12,1)$ \\
Melanoma nodular & $21(9,4)$ \\
Lentigo maligno melanoma & $10(4,5)$ \\
Melanoma polipoide & $9(4)$ \\
Melanoma acral & $8(3,6)$ \\
Melanoma desmoplásico & $4(1,8)$ \\
Melanoma amelanótico & $3(1,3)$ \\
Melanoma associado a nevo & $2(0,9)$ \\
melanocítico & $2(0,9)$ \\
Melanoma pagetoide & $2(0,9)$ \\
Melanoma extensivo superficial com & $1(0,4)$ \\
transformação nodular & $1(0,4)$ \\
Melanoma cutâneo nevoide & $1(0,4)$ \\
Melanoma associado a nevo juncional & $1(0,4)$ \\
\hline $\begin{array}{l}\text { Melanoma associado a nevo } \\
\text { displásico }\end{array}$ & $\mathbf{2 2 4}(100,0)$ \\
\hline Melanoma spitzoide & \\
\hline Total &
\end{tabular}

Entre os 26 pacientes que apresentavam metástase, apenas 24 possuíam registro de sua localização. Os locais mais acometidos por metástases foram linfonodos (50\%), seguidos por pulmáo $(37,5 \%)$, sistema nervoso central (25\%) e fígado $(20,8 \%)$ (Tabela 3$)$.

Tabela 3. Frequência dos locais mais acometidos por metástases

\begin{tabular}{lcc} 
& \multicolumn{2}{c}{ Acometimento } \\
\cline { 2 - 3 } \multicolumn{1}{c}{ Localização } & $\mathbf{n}$ & $\%$ \\
& (total $=\mathbf{2 4})$ & \\
\hline Linfonodo & 12 & 50 \\
\hline Pulmão & 9 & 37,5 \\
\hline Sistema nervoso central & 6 & 25 \\
\hline Fígado & 5 & 20,8 \\
\hline
\end{tabular}

Avaliando o subtipo do tumor em relação à localização corporal, o melanoma extensivo superficial prevaleceu em tronco $(35,6 \%)$, enquanto o nodular e o lentigo maligno acometeram a região cefálica. O lentigo maligno teve quase sua totalidade localizada em áreas fotoexpostas (cabeça e membro superior).

Em relação ao tamanho dos tumores e o subtipo, evidenciou-se que mais de $40 \%$ dos melanomas nodulares apresentavam diâmetro $\geq 2 \mathrm{~cm}$ centímetros ao diagnóstico, enquanto os lentigos malignos e extensivos superficiais foram detectados, em sua maioria, como lesôes clínicas menores. Todos os melanomas nodulares foram classificados como tendo espessura maior ou igual a $1 \mathrm{~mm}$ e $54,6 \%$ tinham Breslow maior ou igual a $4 \mathrm{~mm}$. Já os lentigos malignos e lentiginosos acrais, foram diagnosticados como in situ em $65,7 \%$ e 83,3\%, respectivamente. Os extensivos superficiais entraram na classificação de melanomas finos em mais de $80 \%$ dos casos analisados. Não houve metástases provenientes dos subtipos lentigo maligno e extensivo superficial $(\mathrm{p}<0,001)$. Por outro lado, $80 \%$ das metástases foram provenientes de casos do subtipo "outros melanomas" e $11 \%$ de melanomas nodulares (Tabela 4).

Quando analisadas as características globais dos tumores em relação a indivíduos maiores de 60 anos e menores de 60 anos, observou-se que houve prevalência de lesóes na cabeça e pescoço em indivíduos maiores de 60 anos, ao passo que, em indivíduos menores de 60 anos, a localização mais comum foi o tronco. Em relação ao subtipo das neoplasias, $78 \%$ dos lentigos malignos ocorreram em indivíduos maiores de 60 anos, enquanto o subtipo mais comum em jovens e adultos foram os "outros melanomas" ( $\mathrm{p}<0,001)$. Em relação às metástases, pode-se observar que $85 \%$ ocorreram em indivíduos abaixo dos 60 anos, com significância estatística.

Ao comparar os dados entre os sexos, verificou-se que $70 \%$ das lesōes em membros inferiores e $61 \%$ dos melanomas finos estavam em mulheres. Quanto ao diagnóstico histopatológico, a biópsia excisional foi o procedimento inicial de escolha na maior parte dos tumores, excetuando-se os subtipos acral e lentigo maligno, com relevância estatística. Esse procedimento inicial foi mais comumente utilizado em lesóes menores, com redução das taxas conforme o aumento do tamanho do diâmetro da lesão $(\mathrm{p}=0,449)$.

Associando o subtipo tumoral com índice de Breslow, observou-se que os subtipos mais finos (in situ ou $</=1$ $\mathrm{mm}$ ) foram os extensivos superficiais e lentigo maligno, enquanto os maiores de $1 \mathrm{~mm}$ foram predominantemente nodulares.

\section{DISCUSSÃO}

Esta pesquisa avaliou uma série histórica de melanoma cutâneo de um hospital universitário. No presente Serviço, 
Tabela 4. Associação de subtipos de melanoma com outras características da lesão

\begin{tabular}{|c|c|c|c|c|c|c|c|}
\hline \multirow[b]{2}{*}{ Variável } & \multirow[b]{2}{*}{ Classificação } & \multicolumn{5}{|c|}{ Subtipo de melanoma } & \multirow[b]{2}{*}{$\mathbf{P}$} \\
\hline & & Nodular & $\begin{array}{l}\text { Lentigo } \\
\text { maligno }\end{array}$ & Outros & $\begin{array}{l}\text { Extensivo } \\
\text { superficial }\end{array}$ & Acral & \\
\hline \multirow{6}{*}{ Localização } & $\begin{array}{l}\text { Membro } \\
\text { superior }\end{array}$ & $7(25,0)$ & $6(16,2)$ & $9(13,2)$ & $17(23,3)$ & $1(12,5)$ & - \\
\hline & $\begin{array}{l}\text { Membro } \\
\text { inferior }\end{array}$ & $5(17,9)$ & $4(10,8)$ & $6(8,8)$ & $11(15,1)$ & $1(12,5)$ & - \\
\hline & Tronco & $7(25)$ & $2(5,4)$ & $23(33,8)$ & $26(35,6)$ & $0(0)$ & - \\
\hline & Cabeça & $9(32,1)$ & $25(67,6)$ & $21(30,9)$ & $19(26,0)$ & $0(0)$ & - \\
\hline & Pé & $0(0)$ & $0(0)$ & $0(0)$ & $0(0)$ & $4(50,0)$ & - \\
\hline & Ungueal & $0(0)$ & $0(0)$ & $0(0)$ & $0(0)$ & $1(12,5)$ & - \\
\hline \multirow{4}{*}{ Tamanho $(\mathrm{cm})$} & $\leq 0,5$ & $0(0)$ & $1(7,1)$ & $1(7,1)$ & $4(8,5)$ & $0(0)$ & - \\
\hline & $0,5-1$ & $3(25)$ & $4(28,6)$ & $7(50)$ & $12(25,5)$ & $3(75,0)$ & - \\
\hline & $1-2$ & $4(33,3)$ & $6(42,9)$ & $3(21,4)$ & $26(55,3)$ & $0(0)$ & - \\
\hline & $\geq 2$ & $5(41,7)$ & $3(21,4)$ & $3(21,4)$ & $5(10,6)$ & $1(25,0)$ & 0,794 \\
\hline \multirow{5}{*}{ Breslow (mm) } & In situ & $0(0)$ & $23(65,7)$ & $23(56,1)$ & $21(29,6)$ & $5(83,3)$ & - \\
\hline & $\leq 1$ & $0(0)$ & $9(25,7)$ & $4(9,8)$ & $38(53,5)$ & $0(0)$ & - \\
\hline & $1-2$ & $3(13,6)$ & $0(0)$ & $3(7,3)$ & $7(9,9)$ & $1(16,7)$ & - \\
\hline & $2-4$ & $7(31,8)$ & $1(2,9)$ & $3(7,3)$ & $4(5,6)$ & $0(0)$ & - \\
\hline & $\geq 4$ & $12(54,6)$ & $2(5,7)$ & $8(19,5)$ & $1(1,4)$ & $0(0)$ & - \\
\hline \multirow{2}{*}{ Metástase } & Não & $1(25,0)$ & $22(100)$ & $25(53,2)$ & $25(100)$ & $6(85,7)$ & - \\
\hline & Sim & $3(75,0)$ & $0(0)$ & $22(46,8)$ & $0(0)$ & $1(14,3)$ & $<0,001$ \\
\hline \multirow{2}{*}{$\begin{array}{l}\text { Primeiro } \\
\text { procedimento }\end{array}$} & $\begin{array}{l}\text { Biópsia } \\
\text { excisional }\end{array}$ & $11(84,6)$ & $10(41,7)$ & $16(72,7)$ & $38(76)$ & $2(33,3)$ & - \\
\hline & $\begin{array}{l}\text { Biópsia } \\
\text { incisional }\end{array}$ & $2(15,4)$ & $14(58,3)$ & $5(22,7)$ & $12(24)$ & $4(66,7)$ & 0,006 \\
\hline
\end{tabular}

essa taxa manteve-se estável e possivelmente o pico verificado no ano de 2008 resultou da maior divulgação do tema e da busca de orientação médica decorrente de polêmicas geradas pela Lei que culminou posteriormente com a proibição de bronzeamento artificial no país.

A faixa etária média de 57,3 anos dos pacientes avaliados nesse hospital foi similar a investigações conduzidas no Sul, Sudeste e Centro-Oeste brasileiros ${ }^{5,11-17}$, bem como na América do Norte e Europa ${ }^{18-20}$. No entanto, chama a atençáo que a maior parte da presente casuística encontrava-se em idade produtiva inferior a 60 anos, com possíveis repercussôes socioeconômicas, perda na produtividade e na qualidade de vida.

Discreto predomínio no sexo feminino corrobora achados das literaturas nacional e internacional. Em Minas Gerais, a proporção de mulheres para homens chegou a $1,6: 1^{21}$. Classicamente, as mulheres são mais preocupadas com a saúde, porém há autores que atribuem o fato a essas pacientes utilizarem camas de bronzeamento ${ }^{6}$. A raça branca compreendeu $98,6 \%$ dos casos, sendo explicada pela miscigenaçáo racial regional e também pela maior susceptibilidade da pele clara à agressáo pelos raios ultravioleta. Mesmo nos países com menor prevalência de população branca, esta é ainda a mais acometida ${ }^{1,2,11,14,17,20,22}$.

O aumento de tamanho da lesão como queixa mais prevalente se assemelhou a estudo em Minas Gerais, compreendendo $58,1 \%$ da totalidade ${ }^{21}$. Entretanto, o tempo médio de evoluçáo das lesóes até o diagnóstico foi de 4,8 anos, diferindo de pesquisa realizada em Joinville-SC, na qual essa média de tempo médio foi de 2,2 anos $^{1}$. No estudo catarinense, foram avaliados dados de tumores provenientes de todos os níveis da saúde pública e privada da cidade. Já na presente casuística, o tempo dispendido do trajeto ao diagnóstico é preocupante e pode indicar dificuldade no acesso aos serviços de saúde ou falha no reconhecimento de casos iniciais pela atençáo primária, que poderiam ser beneficiados com a aplicação da regra do ABCDE (assimetria, bordo, cor, diâmetro, elevação), aumentando a detecção de lesóes finas e com 
menor potencial de gerar metástases. Esse fato reforça a necessidade de aprimorar a educação médica continuada, divulgar o autoexame de pele, identificar e corrigir os vazios assistenciais, reduzindo a demora no diagnóstico e tratamento.

$\mathrm{Na}$ análise global, um terço dos tumores localizava-se na cabeça e pescoço, seguido pelo tronco em 27,1\% dos casos. Ao analisar a localização em relação ao sexo, o melanoma de cabeça e pescoço manteve-se prevalente em ambos, no entanto, $70 \%$ das lesôes em membros inferiores acometeram as mulheres. Houve predominância de lesôes na cabeça e pescoço de maiores de 60 anos, ao passo que, em indivíduos menores de 60 anos, a localização mais comum foi o tronco. A literatura mundial mostra predileção do melanoma em tronco nos homens e em membros inferiores nas mulheres ${ }^{5,11,18}$.

Entretanto, ao avaliar estudos regionais, percebe-se que a distribuição étnica e geográfica faz com que esses dados variem muito nas publicaçôes sobre o tema. Em Blumenau-SC, o tronco foi a regiáo mais acometida nas duas populaçôes, já em Joinville-SC, assim como em Curitiba-PR, os homens foram mais acometidos em cabeça e pescoço, enquanto as mulheres tiveram mais lesóes em membros inferiores ${ }^{1,14,23}$. Dessa forma, a análise segmentada dos pacientes atendidos nesse hospital universitário é relevante, uma vez que, pelas características socioeconômicas, é plausível que haja peculiaridades contrastantes com dados provenientes de serviços privados.

Em relação ao tamanho clínico das lesões, 74,8\% possuíam diâmetro de 0,5 a $2 \mathrm{~cm}$, com ligeira predominância das lesões maiores de $1 \mathrm{~cm}$. Apenas $6 \%$ dos tumores eram menores ou iguais a $5 \mathrm{~mm}$. Esse dado vai ao encontro da regra do $\mathrm{ABCDE}$, em que lesóes suspeitas, em teoria, sáo maiores que $6 \mathrm{~mm}$. Quando analisado o tamanho dos tumores em relaçáo ao subtipo, evidenciou-se que mais de $40 \%$ dos melanomas nodulares apresentavam mais de $2 \mathrm{~cm}$ de diâmetro ao diagnóstico, enquanto os lentigos malignos e extensivos superficiais eram detectados como lesôes clínicas menores, em sua maioria entre $1 \mathrm{a} 2 \mathrm{~cm}$. O procedimento inicial na maioria dos pacientes foi biópsia excisional, como é o indicado, exceto nos subtipos lentigo maligno e lentiginoso acral. As prováveis causas para a realização de biópsia incisional ao invés de excisional são a baixa suspeita de melanoma na consulta inicial e a dimensão do tumor, visto que, no presente estudo, observou-se que o aumento do tamanho da lesão foi diretamente proporcional ao uso da técnica excisional.

Em concordância com publicações recentes, o subtipo mais frequente foi o melanoma extensivo superficial $(31,7 \%)$. No entanto, nesta análise, o lentigo maligno teve ligeira prevalência sobre o melanoma nodular e $78 \%$ deles ocorreram em indivíduos maiores de 60 anos de idade. Isso poderia ser explicado pela maior radiação ultravioleta acumulada sofrida por pacientes maiores de 60 anos. "Outros melanomas" tiveram uma prevalência de $34,8 \%$ e ocorreram predominantemente em jovens. Pesquisa realizada em 2013 no Nordeste identificou que mais da metade dos tumores eram do subtipo nodular. Em Minas Gerais, o lentigo maligno foi o mais comum. Esses dados mostram variação de acordo com as características populacionais $^{21,24}$.

A menor prevalência de melanoma acral $(3,6 \%)$, subtipo de melanoma agressivo mais prevalente em indivíduos de pele mais pigmentada, se deve às características demográficas da Região onde o estudo foi realizado, resultado similar a estudo realizado na mesma Região $0^{5,25}$.

Associando-se o subtipo do tumor em relação à localização corporal, corroborando os dados da literatura, o subtipo extensivo superficial prevaleceu em tronco $(35,6 \%)$, enquanto $86 \%$ dos lentigos malignos localizavam-se em cabeça e membros superiores ${ }^{19}$. É provável que o aparecimento dos lentigos malignos em áreas fotoexpostas se deva ao fato de terem a exposição solar crônica como o principal fator de risco para seu surgimento $^{2,5,6,19,26}$. Portanto, medidas fotoprotetoras sistemáticas, individuais e coletivas, precisam ser divulgadas constantemente e incorporadas pela população desde a infância para evitar queimaduras solares e outros danos da exposição desprotegida, como o câncer da pele.

Quase metade dos tumores analisados era restrita à epiderme e 70,2\% foram classificados como melanomas finos $(\leq 1 \mathrm{~mm})$. Em relação ao estadiamento, $83 \%$ dos extensivos superficiais e $91 \%$ dos lentigos malignos eram T1. Esse dado vai ao encontro de estatísticas de países de primeiro mundo, como os Estados Unidos, Suíça e Holanda, onde foram encontrados tumores finos em cerca de dois terços dos pacientes ${ }^{14,18,24}$. Em contrapartida, em estudo realizado por Vilanova et al. ${ }^{9}$, no Nordeste brasileiro, foram encontrados apenas $27,6 \%$ de melanomas finos, com espessura média dos tumores de $8,8 \mathrm{~mm}$ e alta prevalência do subtipo nodular, retratando atraso no diagnóstico e piora no prognóstico dos pacientes ${ }^{2,19,20,24}$.

Ao avaliar o subtipo tumoral em relação índice de Breslow, observa-se, com relevância estatística, que os subtipos mais finos (in situ ou $\leq 1 \mathrm{~mm}$ ) foram os extensivos superficiais e lentigo maligno, ao passo que os maiores de $1 \mathrm{~mm}$ foram predominantemente nodulares. Um estudo suíço com mais de oito mil casos avaliados evidenciou a mesma relação. Além disso, cerca de $25 \%$ dos pacientes que apresentavam subtipo nodular já tinham espessura 
tumoral maior que $4 \mathrm{~mm}$ ao diagnóstico. Esse dado reforça a característica tumoral de agressividade e alerta para a necessidade de sempre documentar o subtipo específico da neoplasia ${ }^{6,14,19}$.

A cirurgia foi o tratamento único de escolha em quase $80 \%$ dos casos da presente casuística. Nos que necessitaram de terapia adjuvante, a quimioterapia teve leve predominância sobre a radioterapia. Dos pacientes que possuíam informaçóes em relação a metástases, $25 \%$ tiveram algum tipo de disseminação interna do tumor primário, semelhante ao estudo nordestino ${ }^{24}$. Pesquisadores de Belo Horizonte-MG, em 2013, no que diz respeito à metástase, encontraram apenas $13,4 \%{ }^{20}$. É possível que esse dado esteja superestimado, visto que não havia informação sobre metástases em mais de 50\% dos registros. Corroborando dados da literatura, a metástase linfonodal foi a mais frequente, seguida por pulmáo e sistema nervoso central. $\mathrm{O}$ acometimento hepático foi o quarto sítio mais comprometido por metástase, em número de casos, podendo inclusive aparecer antes ou depois da metástase cerebral.

Em relação às metástases, houve alta taxa de disseminaçáo do subtipo nodular, enquanto os lentigos malignos e extensivos superficiais não tiveram metástase. No grupo "outros melanomas", 46,8\% apresentaram metástase, apesar de apenas 14, do total de 41 pacientes do grupo, terem Breslow maior de $1 \mathrm{~mm}$ de espessura.

Entre as limitaçôes deste estudo, podem ser elencados o delineamento retrospectivo e a incompletude do banco de dados. Muito embora o prontuário médico seja de preenchimento obrigatório, a quantidade e a qualidade dos seus registros se relacionam a múltiplos fatores humanos $\mathrm{e}$ organizacionais, peculiares aos hospitais públicos de ensino. Todavia, como o melanoma é um problema de saúde pública, a abordagem regional dos casos realizada nesta investigaçáo contribuiu com um conjunto de dados com potencial de ampliar a avaliação do comportamento dessa doença e estimular estratégias educativas e preventivas para diminuir seu impacto e suas consequências.

\section{CONCLUSÃO}

O acometimento de adultos brancos e o predomínio de melanoma extensivo superficial foram similares à literatura regional. A média de 2,99 casos diagnosticados para cada mil atendimentos gerais se manteve estável ao longo dos anos, entretanto, o tempo médio entre o aparecimento da lesão e o diagnóstico, bem como o tamanho do tumor maior de $1 \mathrm{~cm}$, alerta para a necessidade de aprimorar investimentos em estratégias preventivas e terapêuticas. Os melanomas "não clássicos" (subtipos que não sejam extensivo superficial, lentiginoso, lentiginoso acral e nodular) tiveram alta frequência neste estudo, fato que deve chamar atenção para a melhoria dos laudos anatomopatológicos e o aprimoramento de técnicas para diagnóstico precoce e treinamento de profissionais atuantes na atenção básica para reconhecimento de lesôes com apresentação atípica. Tais medidas visam à detecção precoce das lesôes e, consequentemente, à reduçấo da morbimortalidade do melanoma, visto seu impacto social.

\section{CONTRIBUIÇÕES}

Todos os autores contribuíram substancialmente na concepção e/ou no planejamento do estudo; na obtenção, na análise e/ou interpretação dos dados; na redação e/ou revisão crítica; e aprovaram a versão final a ser publicada.

\section{DECLARAÇÃO DE CONFLITO DE INTERESSES}

Nada a declarar.

\section{FONTES DE FINANCIAMENTO}

Não há.

\section{REFERÊNCIAS}

1. Steglich RB, Coelho KMPA, Cardoso S, et al. Epidemiological and histopathological aspects of primary cutaneous melanoma in residents of Joinville, 20032014. An Bras Dermatol. 2018;93(1):45-53. doi: https:// doi.org/10.1590/abd1806-4841.20185497

2. Soong SJ, Ding S, Coit D, et al. Predicting survival outcome of localized melanoma: an electronic prediction tool based on the AJCC melanoma database. Ann Surg Oncol. 2010;17(8):2006-14. doi: https://doi.org/ 10.1245/s10434-010-1050-z

3. Instituto Nacional de Câncer José Alencar Gomes da Silva. Estimativa 2020: incidência de câncer no Brasil [Internet]. Rio de Janeiro: INCA; 2019 [acesso 2020 jul 9]. Disponível em: https://www.inca.gov.br/sites/ufu. sti.inca.local/files//media/document//estimativa-2020incidencia-de-cancer-no-brasil.pdf

4. Ferreira T, Santos IDAO, Oliveira AF, et al. Estudo retrospectivo dos pacientes portadores de melanoma cutâneo atendidos na Universidade Federal de São Paulo. Rev Col Bras Cir. 2018;45(4):e1715. doi: https://doi. org/10.1590/0100-6991e-20181715

5. Purim KSM, Sandri CO, Pinto NT, et al. Perfil de casos de melanoma em um Hospital Universitario, 2003 a 2007. Rev Bras Cancerol [Internet]. 2013 [acesso 2020 abr 9];59(2):193-9. Disponível em: https://rbc.inca.gov. br/revista/index.php/revista/article/view/523

6. Schadendorf D, Fisher DE, Garbe C, et al. Melanoma. Nat Rev Dis Primers. 2015;1:15003. doi: https://doi. org/10.1038/nrdp.2015.3 
7. Ferrari Júnior NM, Muller $H$, Ribeiro $M$, et al. Cutaneous melanoma: descriptive epidemiological study. São Paulo Med J. 2008;126(1):41-7. doi: https://doi. org/10.1590/s1516-31802008000100008

8. Cherobin ACFP, Wainstein AJA, Colosimo EA, et al. Prognostic factors for metastasis in cutaneous melanoma. An Bras Dermatol. 2018;93(1):19-26. doi: https://doi. org/10.1590/abd1806-4841.20184779

9. Vilanova CMA, Lages RB, Ribeiro SM, et al. Perfil epidemiológico e histopatológico do melanoma cutâneo em um centro do nordeste brasileiro de 2000 a 2010. An Bras Dermatol. 2013;88(4):545-53. doi: https://doi. org/10.1590/abd1806-4841.20132036

10. Balch CM, Gershenwald JE, Soong S, et al. Final version of 2009 AJCC melanoma staging and classification. J Clin Oncol. 2009;27(36):6199-6206. doi: https:/doi. org/10.1200/JCO.2009.23.4799

11. Naser N. Cutaneous melanoma: a 30-year-long epidemiological study conducted in a city in southern Brazil, from 1980-2009. An Bras Dermatol. 2011;86(5):932-41. doi: https://doi.org/10.1590/ S0365-05962011000500011

12. Konrad P, Fabris MR, Melao S, et al. Histopathological and epidemiological profile of cases of primary cutaneous melanoma diagnosed in Criciuma-SC between 2005 and 2007. An Bras Dermatol. 2011;86(3):457-61. doi: https://doi.org/10.1590/S0365-05962011000300006

13. Gon ADS, Minelli L, Guembarovski AL. Melanoma cutâneo primário em Londrina. An Bras Dermatol. 2001;76(4):413-26.

14. Ward WH, Farma JM, editors. Cutaneous melanoma: etiology and therapy [Internet]. Brisbane (AU): Codon Publications; 2017. Chapter 6, Clinical presentation and staging of melanoma; [cited 20192019 jun 27]. Available from: https://www.ncbi.nlm.nih.gov/books/ NBK481857/

15. Pinheiro AMC, Friedman H, Cabral ALSV, et al. Melanoma cutâneo: características clínicas, epidemiológicas e histopatológicas no Hospital Universitário de Brasília entre janeiro de 1994 e abril de 1999. An Bras Dermatol. 2003;78(2):179-86. doi: https://doi.org/10.1590/S0365-05962003000200005

16. Muinonen-Martin AJ, O'Shea SJ, Newton-Bishop J. Amelanotic melanoma. BMJ. 2018;360:k826. doi: https://doi.org/10.1136/bmj.k826

17. Wainstein AJA, Duprat Neto JP, Enokihara MY, et al. Demographic, clinical, and pathologic features of patients with cutaneous melanoma: final analysis of the brazilian melanoma group database. JCO Glob Oncol. 2020;6:575-82. doi: https://doi.org/10.1200/ JGO.20.00005

18. Hollestein LM, van den Akker SAW, Nijsten T, et al. Trends of cutaneous melanoma in the Netherlands: increasing incidence rates among all Breslow thickness categories and rising mortality rates since 1989. Ann Oncol. 2012;23(2):524-30. doi: https://doi. org/10.1093/annonc/mdr128

19. Minini R, Rohrmann S, Braun R, et al. Incidence trends and clinical-pathological characteristics of invasive cutaneous melanoma from 1980 to 2010 in the Canton of Zurich, Switzerland. Melanoma Res. 2017;27(2):145-51. doi: https://doi.org/10.1097/ CMR.0000000000000312

20. Pinto ACVD, Cavalcante MLLL, Silva GV, et al. Melanoma maligno: estudo epidemiológico dos casos diagnosticados em unidade de referência em dermatologia em Bauru-sp de 2007 a 2014. Surg Cosmet Dermatol [Internet]. 2015 [acesso 2019 jun 27];7(2):104-7. Disponível em: http://www.surgicalcosmetic.org. br/detalhe-artigo/397/Melanoma-maligno--estudoepidemiologico-dos-casos-diagnosticados-em-unidadede-referencia-em-dermatologia-em-Bauru-sp-de2007-a-2014

21. Gomes J, Parente J, Viana I, et al. Utilidade do HMB-45 e do KI-67 em melanoma maligno associado a nevo. Rev Soc Port Dermatol Venereol. 2011;69(2):195-201. doi: https://doi.org/10.29021/spdv.69.2.605

22. Fernandes NC, Calmon R, Maceira JP, et al. Cutaneous melanoma: prospective study of 65 cases. An Bras Dermatol. 2005;80(1):25-34. doi: https://doi. org/10.1590/S0365-05962005000100004

23. Parente J, Gomes J, Viana I, et al. Variantes raras do melanoma maligno: um desafio clínico e histopatológico. Rev Soc Port Dermatol Venereol. 2012;70(2):195. doi: https://doi.org/10.29021/spdv.70.2.26

24. Foiato TF, Bereza BRK, Montenegro MF, et al. Analysis of patients diagnosed with primary cutaneous melanoma in the last six years in Hospital Erasto Gaertner: epidemiologic profile. An Bras Dermatol. 2018;93(3):332-336. doi: https://doi.org/10.1590/ abd1806-4841.20185788

25. Bradford PT, Goldstein AM, McMaster ML, et al. Acral lentiginous melanoma: incidence and survival patterns in the United States, 1986-2005. Arch Dermatol. 2009;145(4):427-34. doi: https://doi.org/10.1001/ archdermatol.2008.609

26. Marghoob AA, Koenig K, Bittencourt FV, et al. Breslow thickness and Clark level in melanoma: support for including level in pathology reports and in American Joint Committee on Cancer Staging. Cancer. 2000;88(3):589-95. doi: https://doi.org/10.1002/ (SICI) 1097-0142(20000201)88:3<589::AIDCNCR15>3.0.CO;2-I 